\title{
Characterization of dislocation structures and deformation mechanisms in as-grown and deformed directionally solidified NiAl-Mo composites
}

J. Kwon ${ }^{1}$, M.L. Bowers ${ }^{1}$, M.C. Brandes ${ }^{1}$, V. McCreary ${ }^{2}$, I.M. Robertson ${ }^{2}$, P. Sudaharshan

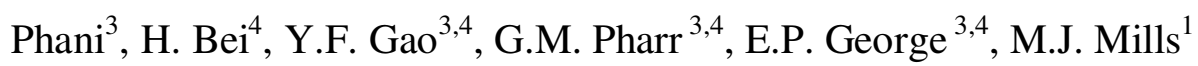

${ }^{1}$ Department of Materials Science and Engineering, The Ohio State University, Columbus, Ohio 43221

${ }^{2}$ Department of Materials Science and Engineering, University of Illinois, Urbana, Illinois 61801

${ }^{3}$ Department of Materials Science and Engineering, The University of Tennessee, Knoxville, Tennessee 37996

${ }^{4}$ Materials Science and Technology Division, Oak Ridge National Laboratory, Oak Ridge, Tennessee 37831

\begin{abstract}
Directionally solidified (DS) NiAl-Mo eutectic composites were strained to plastic strain values ranging from $0-12 \%$ to investigate the origin of the previously observed stochastic versus deterministic mechanical behaviors of Mo-alloy micropillars in terms of the development of dislocation structures at different pre-strain levels. The DS composites consist of long, [100] single-crystal Mo-alloy fibers with approximately square cross-sections embedded in a [100] single-crystal NiAl matrix. Scanning transmission electron microscopy (STEM) and computational stress state analysis were conducted for the current study. STEM of the as-grown samples (without pre-straining) reveal no dislocations in the investigated Mo-alloy fibers. In the NiAl matrix, on the other hand, $a<100>$-type dislocations exist in two orthogonal orientations: along the [100] Mo fiber axis, and wrapped around the fiber axis. They presumably form to accommodate the different thermal contractions of the two phases during cool down after eutectic solidification. At intermediate pre-strain levels (4-8\%), $a / 2<111>$-type dislocations are present in the Mo-alloy fibers and the pre-existing dislocations in the NiAl matrix seem to be swept toward the interphase boundary. Some of the dislocations in the Mo-alloy fibers appear to be transformed from $a<100>$-type dislocations present in the NiAl matrix. Subsequently, the transformed dislocations in the fibers propagate through the NiAl matrix as $a<111>$ dislocations and aid in initiating additional slip bands in adjacent fibers. Thereafter, co-deformation
\end{abstract}


presumably occurs by $<111>$ slip in both phases. With further increase in the pre-strain level ( $>$ $10 \%$ ), multiple $a / 2<111>$-type dislocations are observed in many locations in the Mo-alloy fibers. Interactions between these systems upon subsequent deformation could lead to stable junctions and persistent dislocation sources. The transition from stochastic to deterministic, bulklike behavior in sub-micron Mo-alloy pillars may therefore be related to an increasing number of multiple $a<111>$ dislocation systems within the Mo fibers with increasing pre-strain, considering that the bulk-like behavior is governed by the forest hardening of these junctions.

Keywords: directional solidification, dislocation microstructure, in situ composites, fiber-matrix interaction

\section{Introduction}

As engineering devices have reduced to sub-micron dimensions, it has become increasingly important to quantitatively understand materials behavior at small length scales. Since Uchic et al. [1] first introduced a novel nano-compression testing technique to deform micro-pillars made by focused ion beam (FIB) machining, many experimental studies have shown dramatic size-dependent mechanical behaviors suggesting that "smaller is stronger". Quite remarkably, when sample dimensions are in the micron size range, the material strength becomes stochastic such that it is impossible to precisely predict the stress-strain behaviors of nominally similar specimens [2-5]. Two distinct phenomena have been observed in this stochastic regime: large scatter in flow/yield stress (with average stress values much larger than "bulk" strengths) and strain bursts. This behavior has been explained by various models involving dislocation motion, multiplication, and interactions [3, 5-7].

Recent experiments have shown that size-dependent mechanical behavior is strongly affected by the degree to which pre-existing dislocations are present in the samples [8-9]. In these tests, Mo-alloy micropillars were prepared for compression by selectively etching away the matrix in directionally solidified (DS) NiAl-Mo eutectic composite. The details of the DS process can be found in [10]. As-grown single-crystal Mo-alloy pillars (0\% pre-strain) exhibit the theoretical strength ( 9.3 GPa) independent of sample size. However, when the NiAl-Mo composite is pre-strained to large value $(\sim 11 \%)$ and the exposed Mo-alloy pillars subsequently tested, significant softening $(\sim 1 \mathrm{GPa})$ is observed and bulk-like behavior independent of sample 
size is obtained. Thus, in two extreme cases (no dislocations or many dislocations), the two distinct phenomena mentioned earlier that were believed to be indicative of the small-scale regime were not observed (even in submicron pillars). Only in pillars subjected to intermediate pre-strains (4-8 \%) are features normally associated with small-scale mechanical behavior observed, including an increase in yield strength as the pillar size decreases, stochastic strain bursts, and stochastic yield strengths ranging 1-3 GPa. Bei et al. [8] suggested that the reason for their occurrence only at intermediate pre-strains is that this is when the two characteristic length scales of the problem, dislocation spacing and specimen size, become comparable. In this case, behavior would be strongly dependent on the specific dislocations that are present.

A preliminary TEM analysis on undeformed as well as pre-strained Mo-alloy fibers after chemical extraction from the NiAl matrix has been performed by Phani et al. [11]. In that work, a low dislocation density was found in the as-grown Mo-alloy fibers, while an inhomogeneous distribution of dislocations was reported for pre-strained Mo fibers at intermediate strain levels. Phani et al. [11] suggested two possible mechanisms to explain the inhomogeneous distribution: (a) cracking in the NiAl matrix during the pre-strain that acts as a stress raiser so that the distribution of dislocations may be concentrated near the cracks, and (b) strain softening which will lead to localized deformation such as slip bands. While this previous research has investigated the correlation of dislocation density and dislocation distribution with the observation of stochastic versus deterministic behavior, a detailed understanding of the dislocation substructures is presently lacking.

Recently, Bulatov [12] introduced the concept that dislocation multi-junctions (ternary junctions) can be formed in BCC metals so that the dislocation networks and the mechanical behavior can be affected by this new mechanism. Creation of the multi-junctions consisting of four different $a / 2<111>$-type of dislocations is energetically favorable and may provide strong pinning points and much more stable dislocation nucleation sites than normal binary dislocation junctions. Another possible scenario is that the transmission of dislocations from the NiAl matrix to the Mo fiber could play a role in establishing dislocation distributions inside the fiber. Previous studies have shown the possibility of dislocation transmission in other metal/intermetallic systems [13].

In this study, scanning transmission electron microscopy (STEM) has been used to directly explore these hypotheses and seek evidence for the multi-junction and slip transfer mechanisms 
in the NiAl-Mo fiber eutectic system. The results suggest a direct connection between dislocation configurations and the transitions observed in the mechanical response of micropillars as a function of pre-strain.

\section{Experimental}

\section{Fabrication of pre-strained Mo-alloy micro-fibers and TEM samples}

Directionally solidified NiAl-Mo eutectic composites (Ni-45.5Al-9Mo (at. \%)) were grown in an optical floating zone furnace described elsewhere $[8,10]$. The growth conditions used in this study resulted in [100] single crystal Mo-alloy fibers with approximately square cross-sections and sizes of $500 \mathrm{~nm}$ embedded in [100] single-crystal NiAl matrix. Disks of $\sim 2$ $\mathrm{mm}$ in thickness were cut from the as-grown rods, and subsequently the composites were given engineering pre-strains (percent thickness reductions) ranging from 0 to $16 \%$ parallel to the [100] fiber axis by uniaxial compression in a screw-driven Instron machine.

The NiAl matrix was selectively etched back with a 10 vol. $\% \mathrm{HCl}, 10$ vol. $\% \mathrm{H}_{2} \mathrm{O}_{2}, 80$ vol. $\% \mathrm{H}_{2} \mathrm{O}$ etchant [11] to reveal free-standing Mo fibers (Figure 1). The fibers were then put into an ultrasonic bath with methanol solution to free them from the matrix, followed by deposition of the fibers on the 300 mesh holey-carbon copper TEM grid.

To observe the inter-phase boundary between Mo fiber and the NiAl matrix, longitudinal and transverse (relative to the fiber axes) TEM foils were fabricated from the pre-strained NiAlMo composites. A dual-beam focused ion beam (FIB) system (FEI Helios 600) in combination with an in situ plucking system (Omniprobe ${ }^{\mathrm{TM}}$ ) was utilized. The foils were extracted as close to the center of the directionally solidified rod as possible in order to minimize the region of poorly aligned Mo fibers near its outer surface [10]. For longitudinal TEM foils, regions where the Mo fibers were positioned in well-defined rows were selected to insure that the TEM foils contained multiple fibers, and low energy ion milling was used to reduce surface damage. To prepare the transverse foils, the NiAl-Mo composite was sectioned transversely, ground metallographically up to 4000 grit, and polished with $\sim 0.05 \mu \mathrm{m}$ colloidal silica water suspension in an automatic vibratory machine for 24 hours. Subsequently, TEM foils were extracted by FIB machining. These membranes were plucked and attached to Omniprobe grids before thinning to $<\sim 350 \mathrm{~nm}$ at $30 \mathrm{keV}$ at an incidence angle $\leq 2^{\circ}$ and final thinning at $5 \mathrm{keV}$ at an incidence angle $\leq 5^{\circ}$. Prior 
to TEM examination, samples were argon ion milled first at $900 \mathrm{eV}$ and then at $500 \mathrm{eV}$ in a Fischione 1040 Nanomill@ to remove FIB-induced surface damage.

\section{Microstructure analysis}

An FEI Tecnai F20 was used for TEM analysis at $200 \mathrm{keV}$ in scanning transmission electron microscopy (STEM) mode. Although the size of the Mo fibers on the TEM grid was relatively thick $(\sim 550 \mathrm{~nm})$, the fibers could be directly observed using the STEM mode which gives better resolution for thicker TEM foils [14] while avoiding damage due to ion-beam thinning. Simultaneous bright field (BF) and annular, low angle dark field (DF) imaging was conducted on an off-axis detector at a convergence angle $(\alpha)$ of $5.9 \mathrm{mrad}$. The collection angles $(\beta)$ for $\mathrm{BF}$ and $\mathrm{DF}$ imaging were 0-21.5 and 10.7-61.3 mrad, respectively. Moreover, zone axis BF and DF conditions in STEM enabled imaging of most dislocation content simultaneously [14]. To explore the development of dislocation structures in the Mo-alloy fibers, 3-dimensional (3-D) electron tomogram reconstruction was used in combination with STEM and a standard double tilt holder $\left( \pm 35^{\circ}\right)$. A tilt series of STEM BF micrographs was taken along a Kikuchi band aligned along the main tilt axis of TEM. The BF images were collected with a Gatan image processor, and tracing and labeling of dislocation lines for the 3-D reconstruction was conducted using the University of California, San Francisco Chimera software (ver. 1.5.3).

\section{Results}

The dislocation configurations in the Mo-alloy fibers vary dramatically with increasing plastic strain. In as-grown samples, no dislocations were found in this study. The approximate total length of Mo fibers examined was $3 \mu \mathrm{m}$. At intermediate pre-strains (4-8\% plastic strain), the distribution and configuration of dislocations are inhomogeneous. Some fibers (each typically $3 \mu \mathrm{m}$ long) contain many dislocations and others contain none. At higher pre-strains (> $12 \%$ ), the dislocation content in the Mo fibers tends to be much more evenly distributed. These results are consistent with previous work $[11,15]$, and it is noted that the mechanical behavior [7] and dislocation configurations appear to have a relationship in terms of variations with increasing pre-strain levels [9]. Thus, characterizing dislocation configurations and deformation 
mechanisms are critical to assess the transition from stochastic to deterministic mechanical behavior. In the following sections, the substructures in the composite and extracted fibers are discussed in detail, in both the as-grown and pre-strained conditions.

\section{Substructures in the as-grown composite (0\% pre-strain)}

Insight into the dislocation configurations in the pre-strained fibers requires detailed characterization of the NiAl-Mo composite at several pre-strain levels. This analysis is summarized in the STEM diffraction contrast images of Figures 2 and 3, showing views of both transverse and longitudinal FIB-prepared sections of the as-grown composite. The dark and bright regions in both sets of images are associated with the Mo fibers and the NiAl matrix, respectively. No dislocation content was observed in the Mo fibers, consistent with the work of Phani et al. [11] who made observations over much longer lengths of extracted Mo fibers. Note that the feature indicated as F in Figure 3 is an artifact from the FIB milling process (i.e. a "curtaining" feature) and is not dislocation content in the fiber.

The BF images in Figure 2 are all obtained near the [100] zone, which we define as crystallographic orientation of the fibers. Note that the fibers have roughly square cross-sections with prominent planar facets corresponding to (011) and (01) planes of both the Mo and the $\mathrm{NiAl}$ matrix. This is consistent with a cube-on-cube orientation relationship and fiber morphology described in Bei et al. [10]. Three distinct families of dislocations can be identified based on the four diffraction conditions shown in Figure 2. The dislocations labeled as " 1 " are $a[100]$ dislocations observed as half-loops in the NiAl, wrapping around the fibers. In all four images, segments of these dislocations are observed even though $g \cdot b$ is zero in all cases. Contrast is observed due to non-zero values of $g \cdot(b \times u)$ for these near edge dislocations. Characteristic of this imaging condition is the distinctive, symmetric "double-peak" contrast that becomes invisible when the dislocation line directions are aligned along $g$ (so that the value of $g \cdot(b \times u)$ approaches zero). A schematic representation of these loop-like features is shown in Figure 4. The other two dislocation types ( $a[001]$ labeled as " 2 " and $a[010]$ labeled as " 3 " in Figure 2) are also observed. These dislocations can be distinguished from each other by comparing Figures 2(c) and 2(d): in the former, $a$ [001] dislocation contrast is weak/absent while in the latter, $a[010]$ dislocation contrast is minimized. The $a[010]$ and $a[001]$ dislocations are 
seen generally as shorter segments, indicating that most of this content is aligned roughly parallel to the [100] Mo fiber axes, but with frequent deviations such that much of the content is of mixed character. These $a[010]$ and $a[001]$ dislocations are also shown schematically in Figure 4.

Configurations consistent with the above analysis are found in the longitudinal FIB foil (with a foil normal close to [001]) shown in Figure 3, which provides more insight into the line orientations of the dislocation content that has been illustrated in Figure 4. In Figures 3(a) and $3(\mathrm{~b})$, using the diffraction vectors $g(110)$ and $g(1 \overline{1} 0)$, dislocations in two general orientations can be observed. The dislocations aligned roughly perpendicular to the [100] fiber axis are in contrast in Figure 2(c) for $g(200)$, and go out of contrast in Figure 2(d) for $g(020)$. This contrast behavior and general line orientation are consistent with the $a[100]$ dislocations labeled type " 1 " in Figure 2. Conversely, the dislocations stretching roughly along the fiber axis as seen in Figures 3(a-b) and 3(d), but which are out of contrast in Figure 3(c), are consistent with the $a[010]$ dislocation content labeled as type " 3 " in Figure 2, and the $a[001]$ content labeled as type " 2 " in Figure 2. Note that the latter would be seen in $g \cdot(b \times u)$ contrast in Figure 3(c), which explains the diffuse and double peak contrast seen in some cases.

Importantly, dislocations of the type $a<111>$ are not observed based on the data shown in Figures 2 and 3. For instance, comparing Figures 2(a) and 2(b), it is seen that dislocation types 2 and 3 are all present in both images. If these dislocations were of any $a<111>$-type, then they would yield an invisibility for either $g(0 \overline{1} 1)$ or $g(0 \overline{1} \overline{1})$. However, this is not the case as essentially all dislocation content is observed in both Figures 2(a) and 2(b). In fact, $a<111>$-type dislocations are distinctly associated with the pre-strained condition, as discussed in the next section.

Dislocations of the type $a<011>$ are more difficult to categorically rule-out based on the present data. This dislocation type has only rarely been observed in $\mathrm{NiAl}$ compounds under specific conditions of Ni-rich single crystals after higher temperature deformation along the $<001>$ orientation [16], and thus they are unlikely to be favored in the complex stress state existing in this composite. Indeed, dislocations of the type $a[110]$ and $a[1 \overline{1} 0]$ can be ruled-out since they should be in strong contrast for both $g(0 \overline{2} 0)$ and $g(200)$. However, as discussed above, the same dislocations are not observed in contrast in these in Figures 3(c) and 3(d). Dislocations of the type $a[011]$ and $a[01 \overline{1}]$ would yield an invisibility for either $g(0 \overline{1} 1)$ or $g(0 \overline{1} \overline{1})$. However, 
this is not the case as all prominent dislocation content is observed in both Figures 2(a) and 2(b). Dislocations of the type $a[101]$ and $a[10 \overline{1}]$ can not be ruled-out based on the present data-set. Nevertheless, considering the symmetry of the composite, if $a[110]$ and $a[1 \overline{1} 0]$ dislocations are not present, then likewise $a[101]$ and $a[10 \overline{1}]$ types should also not occur.

It is concluded that the primary dislocations present are indeed all three types of $a<100>$ dislocations, as shown schematically in Figure 4. These dislocations presumably help to accommodate the different thermal contractions of the Mo fibers and NiAl matrix. This hypothesis is explored further in the discussion section. Additional diffraction contrast STEM observations provide information on the nature of the matrix/fiber interface. Figure 5 shows images obtained with the interface inclined in the thin foil relative to the electron beam direction so that the matrix and the fiber are overlapping. A fine, parallel set of fringes can be seen in the region of overlap. These are Moiré fringes rather than interface dislocations since the apparent orientation of the fringes varies with (and is perpendicular to) the operative diffraction vector, as can be seen in Figures 5(a) and 5(b). The insets show the fringes more clearly, and the fringe spacing is $\sim 2.5 \mathrm{~nm}$ for both images. Assuming that these are due to translational Morié fringes, and using the room temperature lattice parameters for both NiAl [17] and Mo [10], the difference in the $g\{101\}$ vectors is expected to be $0.418 \mathrm{~nm}^{-1}$, which corresponds to an expected Moiré fringe spacing of $2.4 \mathrm{~nm}$ [18]. Thus, the observed Moiré fringe spacing is consistent with that expected for an incoherent interface [19]. High resolution imaging at the interface would be necessary to obtain further details about the nature of the interface.

\section{Substructures in pre-strained composites - dislocations within Mo fibers}

Figure 6 shows several lower magnification images of the same region of a FIB-extracted, longitudinal cut sample of the $8.2 \%$ pre-strained NiAl-Mo composite, where several Mo fibers have been captured in the same FIB foil. As before, the darker and lighter regions are Mo fiber and NiAl matrix, respectively. The dislocation configurations in the NiAl matrix are notably different compared to those in the as-grown case. Remarkably, this FIB foil appears to capture a distinct change in the deformation process even on this local level. In order to provide a reference for subsequent presentation of results, the fibers are numbered from 1 to 5 in Figure 6. It appears that in this region the extent of deformation within the fibers, as evidenced by the 
remnant dislocation structures, increases with fiber number. Note that this difference in behavior between fibers 1 to 5 is not due to a significant change in the thickness of the FIB lamellae, or to different overlap between the matrix and the fibers. The three images shown are for the [012] zone axis bright-field condition (Figure 6(a)), where all dislocation content is observed, and two different strong-beam diffraction conditions near the [111] zone axis providing invisibility for dislocation content, enabling a Burgers vector determination (when combined with additional imaging conditions not shown). The strong gradient in deformation is clearly seen when comparing fibers 1 and 2, which are nearly devoid of dislocations, while fibers 3, 4, and 5 have significant (and increasing) dislocation content. Several dislocations of the type $a / 2[11 \overline{1}]$ are seen in fiber 2. A single dislocation slip system is dominant in fibers 3 and 4 , with dislocations of the type $a / 2[111]$ present in fiber 3 and $a / 2[11 \overline{1}]$ predominant in fiber 4 . A second set of slip system is detected in fiber 4 (circled region in Figure 6(c)). These dislocations are of the type $a / 2[1 \overline{1} 1]$. Finally, in fiber 5 , two different slip systems $(a / 2[1 \overline{1} 1]$ and $a / 2[11 \overline{1}])$ are equally activated. Thus, at this modest pre-strain level, there appears to be significant local strain gradients such that quite different $a / 2<111>$ slip system activity can be observed in adjacent fibers. However, it is noted that all four $a / 2<111>$-type dislocations are present in the local region containing fibers $2-5$.

An enlarged region showing fibers 3-5 is shown in Figure $7(a)$ using $g(\overline{110})$ in order to clarify additional details of the dislocation structures within the fibers. A first important detail is that single $a / 2<111>$ dislocations are observed, rather than pairs of dislocations. The unpaired nature of the dislocations is particularly evident in fibers 3 and 4. This feature has implications with respect to slip transmission (or lack thereof) between the NiAl matrix and the fibers, as discussed further below. Second, slip trace analysis indicates that these dislocations lie on (112) planes: $a / 2[11 \overline{1}](\overline{2} 1 \overline{1}), a / 2[111](\overline{2} 11), a / 2[1 \overline{1} 1](21 \overline{1})$, and $a / 2[\overline{1} 11](211)$. The dislocation line directions have also been determined based on tilting experiments and line trace analysis. For instance, in Figure 7(a), the foil has been tilted to an asymmetric orientation, as indicated by the stereographic projection (see inset). The projections of the $[11 \overline{1}]$ and $[1 \overline{1} 1]$ line directions are indicated in Figure 7(a). Note that these two directions make different angles relative to the fiber axis. It is clear that these directions coincide with the predominant line directions of the 
$a / 2[11 \overline{1}]$ and $a / 2[1 \overline{1} 1]$ dislocations, indicating that these dislocations have predominantly screw character. Similarly, the $a / 2[111]$ dislocations in fiber 3 are also near-screw orientation. In addition, there are only a few debris loops visible in fibers 3 and 4 (see Figure 7(a)). Since there is only one dislocation type present in these fibers, these small loops are likely to form due to multiple cross-slip events, and the jogs thereby formed along the moving screw dislocations.

Fiber 5 is of particular interest in that it shows clear evidence of slip transfer between the matrix and Mo fiber. Some of dislocations in the slip bands are straight - similar to those in fiber 4 - while others look more tortuous. In addition, many small dislocation loops with diameters less than $10 \mathrm{~nm}$ are observed within the slip bands. Note that there is a significantly greater density of debris loops in fiber 5, as shown in Figure 7(b), compared with that seen in the other fibers, indicating that the higher dislocation density in the shearing locations promotes cross slip events and increased production of debris loops.

\section{Substructures in pre-strained composites - dislocations in the NiAl matrix}

There are significant changes to the dislocation structures in the matrix when comparing different fiber neighborhoods, as shown in Figure 8, which is an overall view of the $8.2 \%$ prestrain sample shown in Figures 6 and 7, but with contrast optimized for viewing the dislocation structures in the NiAl matrix in this zone axis image. From a $g \cdot b$ analysis (details not shown here), all three pre-existing <100>-type dislocations are found in the various regions; however, their distribution relative to the fibers varies significantly. The NiAl matrix above fiber 1, which apparently has experienced a relatively small amount of local deformation based on the absence of dislocations in fiber 1, appears similar to the as-grown structure presented in Figures 2 and 3. There is substantial $a[010]$ and $a[001]$ dislocation content (dislocation lines roughly parallel to the fiber axis) in the center of the $\mathrm{NiAl}$ matrix region. It is noteworthy that adjacent to fibers 2 and 3, which are apparently more deformed, there is far less $a[010]$ and $a[001]$ dislocation content in the center of the matrix regions. Thus, these dislocations seem to have been "swept" toward the fiber interfaces. This is particularly evident at the arrowed locations. In addition, the $a[100]$ dislocations have become more curved and wavy compared to those in the as-grown 
sample (Figure 3). These observations suggest the build-up of stress concentrations at the fiber/matrix interfaces.

It is often found that the $a<111>$-type dislocations traversing the fiber appear to take on a hairpin-like shape (Figure 9) and on occasion can be seen to extend towards the other side of the fiber. It is noted that in this case (fiber 4), many of these hairpin loops are generated in regions where only $a<100>$ dislocation content is present at the interfaces. This is particularly apparent on the "top side" of fiber 4 . This suggests that $a<111>$ activity in the fiber has originated from stress concentrations from the $a<100>$ matrix dislocations. Thus, this is an example where the Mo fiber has locally yielded on this slip system, but this activity did not initiate slip on the matching system in the matrix.

A striking change in deformation mode is observed in the matrix just below fiber 4 and across fiber 5, as seen in Figure 8. While there remains $a<100>$ dislocation content, there are additionally $a<111>$ dislocations that are straight and inclined at distinct angles to the [100] fiber axis. Two different $a<111>$ dislocation slip systems are active, and these have the same Burgers vector direction as the corresponding $a / 2<111>$ dislocations seen in the adjacent Mo fibers. Indeed there appears to be direct connection between $<111>$-type dislocations in the fiber and in the matrix, as evidenced in Figure 10(a). It is seen that $a / 2[11 \overline{1}]$ dislocations in the Mo fibers are connected to <111>-type dislocations in $\mathrm{NiAl}$ across the interphase boundary. Note that an attempt has not been made to determine the magnitude of these Burgers vectors, which is a challenging task for diffraction contrast imaging. However, it is expected that the $<111>$-type dislocations in the $\mathrm{NiAl}$ are indeed dislocations of the type $a<111>$ since these are perfect dislocations in the B2 lattice. The zone axis DF image of Figure 10(b) shows the large local strain concentrations developed in the matrix at the interface with fiber 5 . The associated stress concentrations presumably promote dislocation activity in these locations within the Mo fibers. Note that we observe $a<111>$ activity in the $\mathrm{NiAl}$ matrix only in regions adjacent to where the Mo fibers have been intensively sheared by $a / 2<111>$ slip (in other regions of the NiAl matrix, the dislocations are of the $a<100>$-type). Subsequently, the transformed $a<111>$ dislocations propagate through the $\mathrm{NiAl}$ and aid in initiating additional $a / 2<111>$ slip bands in the adjacent fiber. Thereafter, co-deformation presumably occurs by <111> slip in both phases. Further evidence in support of the sequence of activity proposed above is provided by the singular 
observation of $a / 2[1 \overline{1} 1]$ activity in fiber 4 (indicated by the circled region in Figure 6(c)). This slip system has been activated in the Mo fiber, without any evidence for corresponding $a[1 \overline{1} 1]$ activity in the adjacent NiAl matrix. Thus, this is an example of local yielding of the Mo fiber on this slip system, but the activity has not yet initiated slip on the matching system in the matrix. Further details of the deformation scenario in the NiAl-Mo eutectic system will be described in the discussion section in conjunction with elastic analysis.

Composites pre-strained to smaller values $(2.2 \%$ and $4 \%)$ were also studied. Observations over significant lengths of these fibers indicate that the predominant dislocation content is similar to that seen in fibers 1 through 4 in Figures 6 and 7. Specifically, the fiber substructures are dominated by regions with single families of $a / 2<111>$ dislocations, and these tend to be distributed much more inhomogeneously than those in the $8.2 \%$ pre-strained foil. These results support the hypothesis that the $8.2 \%$ pre-strained foil described above captures a significant local strain gradient, with smaller local strain represented at the top and larger strains at the bottom of the foil. Possible causes for such a large local strain gradient are discussed below.

\section{Dislocation Interactions in $16 \%$ Pre-strained Mo fibers}

Examples of detailed analyses of dislocation structures observed in two different regions of extracted Mo fibers for a $16 \%$ pre-strained composite are now described. A 3-D tomogram reconstruction, as shown in Figure 11, provides additional insight into possible dislocation interactions within the Mo fibers. The images show a region with relatively small dislocation density where all the dislocations have the same $a / 2<111>$-type Burgers vector. It is noted that there are debris loops also present (Figure $11(\mathrm{~d})$ ). In addition, some dislocations appear to interact with their neighbors and become wavy and tortuous, presumably due to strong elastic interactions. Thus, the debris loops appear to be a natural consequence of $a / 2<111>$ slip, and may be enhanced as the dislocation density in a given region increases. Note that the more strongly sheared regions in the $8.2 \%$ pre-strained composite (e.g. fiber 5 in Figure 7(b)) also exhibit a high density of these small debris loops. 
In contrast to the cases described above where a single slip system is operative, several additional regions of fibers have been examined that exhibit multiple dislocations coexisting at larger pre-strain. One such case is shown in Figure 12, which is a fiber from a $16 \%$ pre-strained sample. Due to some surface contamination, there is a contrast difference from left to right along the fiber. This does not, however, reflect a difference in fiber thickness. Figure 12(a) shows all of the dislocations, while the invisibilities of the three $a / 2<111>$-type dislocations are shown in Figure $12(b, c)$. An extensive TEM analysis revealed that only two types of dislocations $(a / 2[1 \overline{1} 1]$ and $a / 2[\overline{1} 11])$ are present in the region analyzed, in spite of the much more complex dislocation line configurations observed here compared to those at smaller pre-strain.

The dislocation structures in the $16 \%$ pre-strained Mo fiber is noticeably different from the substructures in the $8.2 \%$ pre-strained Mo. In addition to the above deformation mechanism, presumably the slip bands of the type seen in fiber 5 in the $8.2 \%$ pre-strained Mo fiber (Figure 7 (b)) further expand along the lateral length of the fiber with additional deformation. While it might be expected that simple binary junctions might be produced from the interaction of two $a / 2<111>$-type dislocations to yield $a<100>$ reaction products, no evidence of such direct reactions have been obtained in the present study. It is certainly possible that such reactions occur upon subsequent deformation of pre-strained Mo-alloy micropillars since the local conditions for such reactions are clearly present.

\section{Discussion}

In the NiAl phase in the as-grown condition, all three types of $a<100>$ dislocations are present, with near-edge character being a prominent line direction. A simulation performed by Glatzel et al. [20] showed that $a<100>$ dislocations are stable in NiAl. Since the coefficients of thermal expansion of the two phases in the NiAl-Mo composite are different [21], introducing $a<100>$ dislocations in the NiAl matrix during cooling would relieve the thermal contraction mismatch. The $a<100>$ dislocation arrangements observed in the as-grown eutectic can be explained from the residual stresses that arise from the thermal contraction mismatch in the Mo fiber and NiAl matrix [21-24]. The thermal expansion coefficient of Mo is less than that of NiAl, so upon cooling from the eutectic temperature, the Mo phase will be subjected to compression and NiAl to tension in the fiber growth direction (illustrated in Figure 13(a)). When the resulting 
stress is large enough, interface dislocations may form to accommodate this mismatch. Furthermore, under the action of a tensile stress along [100] during the cool down after solidification, the $a[100]$ dislocations could climb away from the interfaces, leading to the formation of the $a[100]$ dislocation loop segments seen in Figure 2.

The source of stress responsible for the $a[010]$ and $a[001]$ dislocations requires additional analysis. As shown in Figure 14, the stress states in the fiber-matrix system can be obtained from the Lamé solution of two cylinders with a mismatch strain. The stress components in the matrix are shown. From the method in Budiansky et al. [22], assuming isotropic elasticity and the same Poisson's ratio, $v$, for the fiber and matrix phases, the stresses in $\mathrm{NiAl}$ can be calculated as

$$
\sigma_{r r}=\frac{a^{2}}{R^{2}-a^{2}}\left(1-\frac{R^{2}}{r^{2}}\right) q, \sigma_{\theta \theta}=\frac{a^{2}}{R^{2}-a^{2}}\left(1+\frac{R^{2}}{r^{2}}\right) q
$$

where the interface pressure is $q=\frac{E_{m}}{2 \lambda_{1}}\left(\frac{c_{m}}{1-v}\right)\left(\alpha_{f}-\alpha_{m}\right) \Delta T, \lambda_{1}=1-\frac{1}{2}\left(\frac{1-2 v}{1-v}\right)\left(1-\frac{E}{E_{f}}\right)$, $E=c_{f} E_{f}+c_{m} E_{m}, c_{f}$ and $c_{m}$ are volume fractions of fiber and matrix respectively, $\alpha_{f}$ and $\alpha_{m}$ are their respective thermal expansion coefficients, and $\Delta T(<0)$ is the temperature drop. From Figure 14, we have $c_{f}=a^{2} / R^{2}$. The compressive radial stress and tensile hoop stress will give rise to shear stresses in the $(r, \theta)$ plane that are responsible for the formation of $a[010]$ and $a[001]$ dislocations in NiAl, illustrated in Figure 2. We also note that the stress state in the fiber phase is equi-biaxial in the $(r, \theta)$ plane.

For the pre-strained samples, based on these experimental observations, the following deformation scenario is proposed (Figure 15). It is assumed that the NiAl matrix deforms first because of the pre-existing $<100>$-type dislocations in the as-grown sample as well as the ultrahigh strength of the dislocation-free Mo fibers ( 9 GPa based on [9, 25]). The $a[010]$ and $a[001]$ dislocations in the NiAl matrix accumulate at the interface (e.g. arrows in Figure 8) and create local stress concentrations that activate $a / 2<111>$ slip in the Mo fibers. The presence of $a$ [100] dislocations also at the interfaces implies a more complex process involving glide of non-edge content, perhaps driven by elastic interactions with the $a[010]$ and $a[001]$ dislocations. Next, the $<111>$ slip in the fibers triggers <111>-type dislocations in the NiAl matrix. This sequence of events is supported by the fact that $<111>$-type slip in fibers 1-3 (in Figures 6-8) is observed 
even though no $<111>$-type slip activity is seen in the adjacent matrix, and the fact that $<100>$ type dislocations are the most commonly observed in deformed $\mathrm{NiAl}$ [20]. A possible reason for the accumulation of $<100>$-type dislocations at the interfaces is now discussed.

As illustrated in Figure 13(a), in the as-grown state, the Mo fiber is under compression along its axis and $\mathrm{NiAl}$ under tension because of the thermal contraction mismatch. No dislocations are found in Mo, presumably due to its high strength. Three types of $<100>$ dislocations are found in $\mathrm{NiAl}$, whose presence we attribute (as discussed earlier) to the thermal residual stress. Barabash et al. [24] used synchrotron X-rays to carefully examine the change of residual stresses after pre-strain. Their key findings are summarized in Figure 13(b). Regardless of the thermal residual stresses in the as-grown state, large pre-strains will lead to compressive plastic deformation in both phases. It should be noted that the Mo fiber, despite its high yield strength in dislocation-free states, has a lower strength-to-modulus ratio than the $\mathrm{NiAl}$ phase; that is, its strain at yielding is lower than that of NiAl. Upon unloading, the composite is essentially subjected to an isostrain condition, and the residual stresses parallel to the fiber axis should be opposite in the two phases to maintain force balance. These conditions naturally lead to tensile residual stress in Mo and compression in NiAl, as shown in Figure 13(c). Similarly, the hoop and radial stresses will change signs accordingly.

Because of the sign change of the residual stresses in the NiAl phase, the driving forces on the existing $a<100>$ dislocations, which were driven by the thermal residual stresses in Figure 13(a), will be reversed so that these dislocations will move towards the interface during prestraining. Note that since the unloading step only leads to elastic deformation, it is presumed that the $a / 2<111>$ dislocations in Mo were activated during the pre-straining step. These dislocations will likely accumulate near the Mo-NiAl interface and drive the initiation of $a<111>$ dislocations in the neighboring NiAl phase. Subsequently, during the unloading step, the reversal of the residual stress will drive these $\mathrm{NiAl} a<111>$ dislocations back to the Mo-NiAl interface, as evidenced in Figure 8, and also cause run-back of $a / 2<111>$ hairpin-like features in the Mo fibers, as seen for instance in Figure 9.

An important issue that has been addressed in the present work is whether there may be something fundamentally different about the nature of dislocation configurations in Mo fibers of similar size that are pre-strained to different levels, that may shed additional light on the source of the "stochastic" versus "deterministic" behavior. Recently, Phani et al. [26-28] have 
developed a statistical model that is able to quantitatively explain the transition from ideal, to stochastic, to bulk behavior in the Mo-alloy microcystals. In their model, they utilize not only the observed dislocation density increase as a function of pre-strain, but also assume a random distribution of Schmid factors for these dislocations. The latter assumption is a convenient means of accounting for operation of different slip systems, as well as local configurations that may alter the stresses required for operating dislocation sources. Indeed, the present work has shown that there are clear differences in dislocation content, even for the same pre-strain value, as shown in Figures 6 and 7. For instance, fiber 3 and most of fiber 4 appear to contain only a single set (Burgers vector type) of well separated, near screw $a / 2<111>$ dislocations. For this configuration, subsequent deformation of the Mo fiber could lead to movement of these dislocations to the fiber surfaces, but with potentially little or no persistent activation of sources (similar to what has been seen before during in situ deformation of Mo-alloy fibers in a TEM [29]). On the other hand, the multi-slip configurations observed in fiber 5 exhibiting interactions, cross slip and debris loops, could create local conditions that make persistent source activation and "bulk-like" behavior much more likely. Thus, it is possible that the random distribution of Schmid factors assumed by Phani et al. [27] may have its physical origin in the clearly distinct, local configurations such as those identified in the present work. A more quantitative assessment of the behavior expected for various dislocation configurations would require detailed 3-D dislocation dynamics simulations.

Our results demonstrate that the slip transfer mechanism dominates plastic deformation at intermediate pre-strain levels $(<10 \%)$, which is the strain range that corresponds to the regime where stochastic mechanical behavior is observed in micropillar compression. The stochastic nature can be attributed to the inhomogeneous distribution of (a) dislocation density and (b) dislocation interactions in different locations in the Mo fibers. These inhomogeneities in density and detailed substructure may originate from the randomly accumulated stress raisers at the fiber interfaces. The irregularity of the inter-phase boundary presumably is caused by local imperfections such as growth defects or cracks during the compression test [11], or caused by poor alignment between the platen in the compression tester and the top of the sample surface. Thus, a deformation gradient is inherently present in the composite which can be directly correlated to the stochastic mechanical behaviors. At lower pre-strain, the deformation gradient is significantly high such that the strength values are highly scattered, and as the pre-strain 
increases and co-deformation of the two phases starts to occur, the dislocation distribution becomes more homogeneous so that the deviations of strength values diminish.

Surprisingly, evidence for actual reactions between two active $a / 2<111>$ dislocations in the Mo fibers was not obtained at larger pre-strain values. These reactions that would nominally produce $a[100]$ dislocations (having Burgers vectors parallel to the fiber axis) should be energetically favorable in terms of lowering the local dislocation strain energy, but may only occur upon subsequent deformation of the free-standing Mo pillars. If such reaction products are formed, they could act to "anchor" dislocation sources in the small volume fibers and promote the persistent dislocation sources needed to obtain a deterministic, bulk-like response.

\section{Conclusions}

The origin of the stochastic and deterministic mechanical behaviors of pre-strained Moalloy micropillars produced from the directionally solidified NiAl-Mo eutectic system have been explored in terms of the detailed dislocation configurations that develop during pre-straining. In the as-grown state ( $0 \%$ pre-strain), STEM diffraction contrast analysis reveals no dislocations in the Mo fibers and $a<100>$-type dislocations in the NiAl matrix. With increasing pre-strain level, $<111>$ slip activates in the Mo fibers inhomogeneously. Slip in the Mo fibers appears to initiate from build-up of stress due to the pre-existing <100>-type dislocations in the NiAl matrix, which apparently are swept towards the interphase boundaries during pre-straining. The $<111>$ slip in the Mo fibers is subsequently transmitted to the NiAl matrix where it assists in introducing additional $<111>$ slip activity in the neighboring fibers. At moderate pre-strain levels (e.g. 8.2 $\%)$, Mo fibers can incorporate single, separated $a / 2<111>$ screw dislocations, as well as more complex interactions of multiple slip systems. At higher pre-strain levels, multiple dislocation types are present in the same region of the Mo fibers, making interactions during subsequent deformation more likely. Thus, in addition to dislocation density distribution, the detailed dislocation configurations generated during pre-straining may play a key role in determining the transition from stochastic to deterministic (bulk-like) behavior as a function of the level of prestrain. 


\section{Acknowledgments}

This research was supported by the Center for Defect Physics, an Energy Frontier Research Center, supported by the U.S. Department of Energy, Office of Science, Basic Energy Sciences.

Figure 1. TEM sample preparation procedures from the as-grown and the pre-strained NiAl-Mo composites using both selective etching and FIB extraction.

Figure 2. BF STEM diffraction contrast images from a (100) transverse section of the as-grown NiAl-Mo composite showing analysis of the dislocation content in the NiAl matrix. All images have been obtained near the [100] zone axis and show portions of $a$ [100] dislocation loops encircling the Mo fibers (labeled as " 1 ”). These loops exhibit $g \cdot(b \times u)$ contrast. In (a) and (b), $g(0 \overline{1} 1)$ and $g(0 \overline{1} \overline{1})$ images, respectively, show that other dislocation content (labeled as "2" and " 3 ") is also present. Dislocations in strong contrast in (c) for $g(0 \overline{2} 0)$ (labeled as " 3 ") are out of contrast in (d) for $g(002)$, consistent with $a[010]$ dislocations. Dislocations in strong contrast in (d), labeled as " 2 ", are out of contrast in (c), consistent with $a$ [001] dislocations.

Figure 3. BF STEM diffraction contrast images from a (001) longitudinal section of the as-grown NiAl-Mo composite showing analysis of the dislocation content in the NiAl matrix. In (a) and (b), $g(110)$ and $g(1 \overline{1} 0)$ images, the same dislocation content is observed, which rules out the presence of $a<111>$ dislocations. In (c) with $g(200)$, dislocation content largely perpendicular to the fibers is in contrast, consitent with $a$ [100] dislocations. In (d) for $g(020)$, dislocation content largely parallel to the fibers is in contrast, consitent with $a[010]$ or $a[001]$ dislocations. The Mo fibers exhibit no dislocation content (the feature labeled as " $F$ " is a curtaining artifact from FIB preparation).

Figure 4. Schematic representation of dislocation configurations and stress states in the Mo fiber and the NiAl matrix. Large arrows indicate stress state in the sample; compression in the Mo fiber and tension in the NiAl matrix.

Figure 5. BF STEM images showing Moiré fringe which is an array of diagonal lines at the interphase boundary. The direction of the fringe pattern varies with different $<110>$-type reflections near [111] zone axis. (a) Diagonal fringe pattern running from bottom right to top left and (b) pattern running from bottom left to top right. Enlarged images in top-right corners of 
each image show the Moiré fringe more clearly. The fringe spacing is $\sim 2.5 \mathrm{~nm}$ for both patterns. For scale, the inset images are $50 \mathrm{~nm}$ wide in (a) and $75 \mathrm{~nm}$ wide in (b).

Figure 6. BF STEM images of the $8.2 \%$ pre-strained NiAl-Mo composite exhibiting a significant gradient in deformation. Fibers are numbered from 1 to 5 to provide a reference for subsequent presentation of results. (a) Zone axis ([012]) image depicts all the dislocations in the NiAl matrix and Mo fiber. Dislocation invisibilities in the Mo fibers near the zone axis [111]. (b) Dislocations running from the bottom-right to the top-left corner $(b=a / 2[1 \overline{1} 1])$ are invisible, in (c) the other dislocations $(b=a / 2[11 \overline{1}])$ running primarily from the bottom left to top right are invisible.

Figure 7. (a) Tilted crystal image showing three different $<111>$-type dislocations in the Mo fibers. The dashed white arrows indicate their projected line directions. Although $b=a / 2[1 \overline{1} 1]$ dislocations are under invisible condition, the orientation of the slip bands can still be discerned. The stereographic projection shows the projected Burgers vector directions, indicating that most dislocations have near-screw orientation. (b) Zone axis image illustrating small dislocation debris loops along the $<111>$ slip bands.

Figure 8 . Zone axis image illustrating an overall view of the $8.2 \%$ pre-strained sample shown in Figure 6 and Figure 7, but with optimized contrast for viewing the dislocation structures in the $\mathrm{NiAl}$ matrix. The arrows indicate evidence of $<100>$-type dislocations accumulated near the interfaces.

Figure 9. BF zone axis image illustrating the hairpin-like dislocations (marked with arrows) which can be nucleated by stress accumulation at the interphase boundary.

Figure 10. BF and DF STEM images showing dislocation activities at the interphase boundary

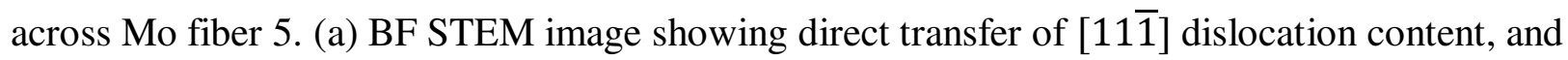
(b) DF zone axis image showing stress build-up at the interface (lighter region) whose locations correspond to the locations where the slip bands in Mo were observed.

Figure 11. (a-c) Representative BF STEM images in a tilt series for 3-D tomogram reconstruction. The relative tilt angles are indicated in each figure. (d) A snap shot from a 
retraced movie. Note that this represents a relatively undeformed region is selected in a $16 \%$ pre-strained Mo fiber.

Figure 12. BF STEM micrographs showing a region in the $16 \%$ pre-strained Mo fiber exhibiting two dislocation families and complex dislocation morphologies. (a) BF image for $g(1 \overline{1} 0)$ refection in which all dislocation content is visible. (b) $g(01 \overline{1})$ image in which $b=a / 2[\overline{1} 11]$ dislocations are invisible, and (c) $g(10 \overline{1})$ image in which $b=a / 2[\overline{1} 11]$ dislocations are invisible. Note that the contrast difference across the fiber is due to some surface contamination of the sample and not due to thickness variation.

Figure 13. The residual stress fields in the composite are reversed due to the large degree of prestraining. Refer to the text and Ref. [24] for interpretations.

Figure 14. The stress states in the fiber-matrix system obtained from the Lamé solution of two cylinders with a mismatch strain. The stress components in the matrix are shown.

Figure 15. Proposed deformation scenario (slip transfer mechanism) for the NiAl-Mo composite. 


\section{References}

[1] M.D. Uchic, D.M. Dimiduk, J.N. Florando, and W.D. Nix, Materials Research Society Symposium Proceedings, 2003. 753: p. 27-32.

[2] J.R. Greer and W.D. Nix, Physical review B, 2006. 73: p. 245410.

[3] M.D. Uchic, D.M. Dimiduk, J.N. Florando, and W.D. Nix, Science, 2004. 305(5686): p. 986989.

[4] M.D. Uchic and D.M. Dimiduk, Materials Science and Engineering A, 2005. 400-401: p. 2068-278.

[5] J.R. Greer, W.C. Oliver, and W.D. Nix, Acta Materialia, 2005. 53: p. 1821-1830.

[6] C.R. Weinberger and W. Cai, Proceedings of National Academy of Sciences, 2008. 105: p. 14304-14307.

[7] A.S. Schneider, B.G. Clark, C.P. Frick, P.A. Gruber, and E. Arzt, Materials Science and Engineering A, 2009. 508: p. 241-246.

[8] H. Bei, S. Shim, G.M. Pharr, and E.P. George, Acta Materialia, 2008. 56: p. 4762-4770.

[9] H. Bei, S. Shim, E.P. George, M.K. Miller, E.G. Herbert, and G.M. Pharr, Scripta Materialia, 2007. 57: p. 397-400.

[10] H. Bei and E.P. George, Acta Materialia, 2005. 53: p. 69-77.

[11] P.S. Phani, K.E. Johanns, G. Duscher, A. Gali, E.P. George, and G.M. Pharr, Acta Materialia, 2011. 59: p. 2172-2179.

[12] V.V. Bulatov, L.L. Hsiung, M. Tang, A. Arsenlis, M.C. Bartelt, W. Cai, J.N. Florando, M. Hiratani, M. Rhee, G. Hommes, T.G. Pierce, and T.D. de la Rubia, Nature, 2006. 440(27): p. 1174-1178.

[13] Y.X. Chen, C.Y. Cui, J.T. Guo, and D.X. Li, Materials Science and Engineering A, 2004. 373: p. 279-285.

[14] P.J. Phillips, M.J. Mills, and M.D. Graef, Philosophical Magazine, 2011. 19: p. 2081-2101. [15] A. Misra, Z.L. Wu, M.T. Kush, and R. Gibala, Materials Science and Engineering A, 1997. 239-240: p. 75-87.

[16] M.J. Mills, R. Srinivasan, and M.J. Daw, Philosophical Magazine A, 1998. 77: p. 801-823. [17] A. Taylor and N.J. Doyle, Journal of applied Crystallography, 1972. 5: p. 210-215.

[18] D.B. Williams and C.B. Carter, Transmission Electron Microscopy. Second ed. 2009, New York: Springer.

[19] J.W. Matthews, Philosophical Magazine, 1966. 13(126): p. 1207-1221.

[20] U.G.K.R. Forbes and W.D. Nix, Philosophical Magazine A, 1993. 67(2): p. 307-323.

[21] H. Bei, E.P. George, D.W. Brown, G.M. Pharr, H. Choo, W.D. Porter, and M.A.M. Bourke, Journal of Applied Physics, 2005. 97: p. 123503.

[22] B. Budiansky, J.W. Hutchinsion, and A.G. Evans, Journal of Mechanics and physics of solids, 1986. 34: p. 167-189.

[23] R.I. Barabash, H. Bei, Y.F. Gao, and G.E. Ice, Scripta Materialia, 2011. 64: p. 900-903.

[24] R.I. Barabash, H. Bei, Y.F. Gao, G.E. Ice, and E.P. George, Journal of Materials Research, 2010. 25: p. 199-206.

[25] H. Bei, Y.F. Gao, S. Shim, E.P. George, and G.M. Pharr, Physical Review B, 2008. 77.

[26] J.R. Morris, H. Bei, G.M. Pharr, and E.P. George, Physical Review Letters, 2011. 106: p. 165502.

[27] P.S. Phani, K.E. Johanns, E.P. George, and G.M. Pharr, Acta Materialia, 2013. 61(7): p. 2489-2499. 
[28] T.L. Li, H. Bei, J.R. Morris, E.P. George, and Y.F. Gao, Materials Science and Technology, 2012. 28: p. 1055-1059.

[29] C. Chisholm, H. Bei, M.B. Lowry, J. Oh, S.A.S. Asif, O.L. Warren, Z.W. Shan, E.P. George, and A.M. Minor, Acta Materialia, 2012. 60(5): p. 2258-2264. 


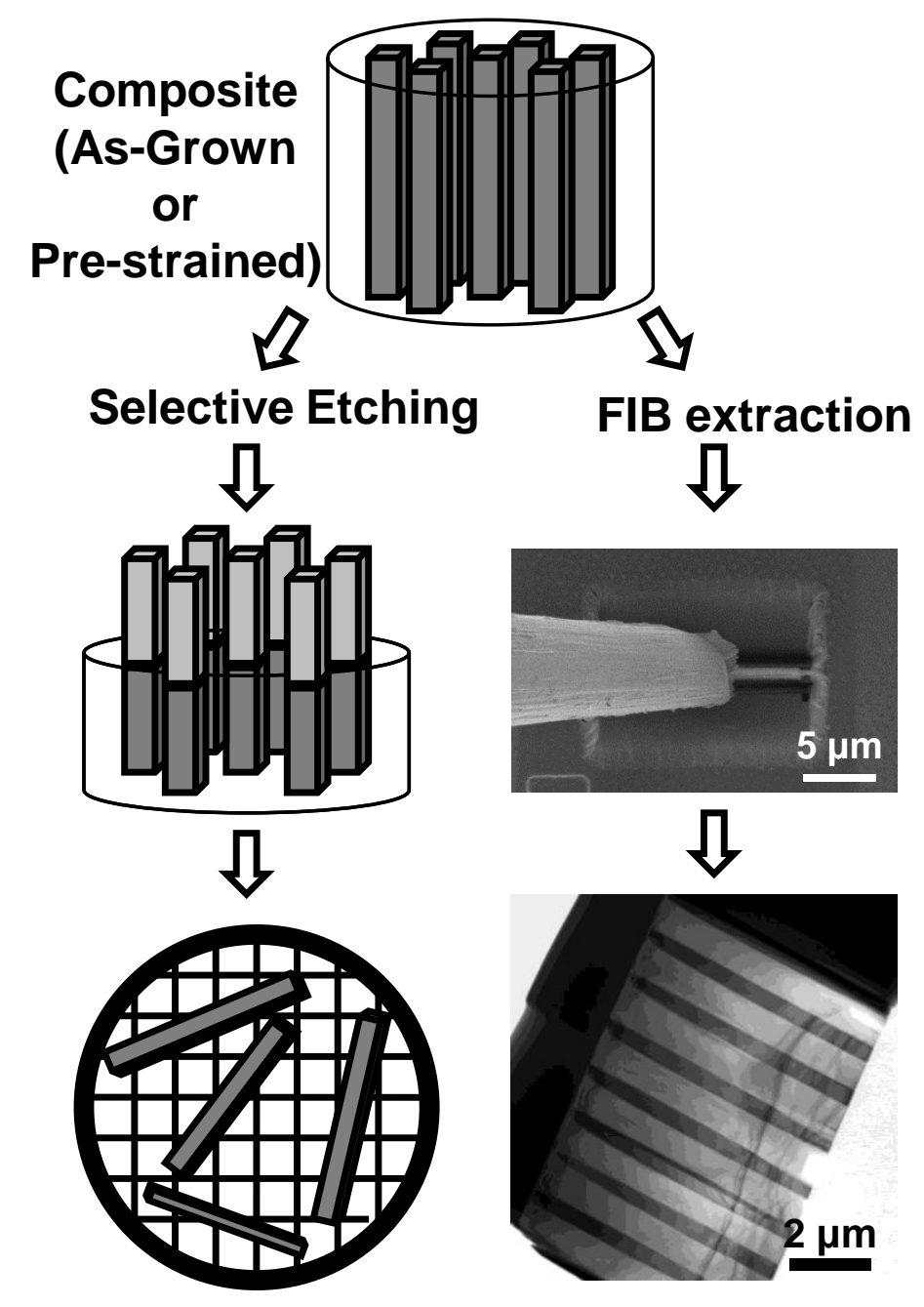

Fig. 1 

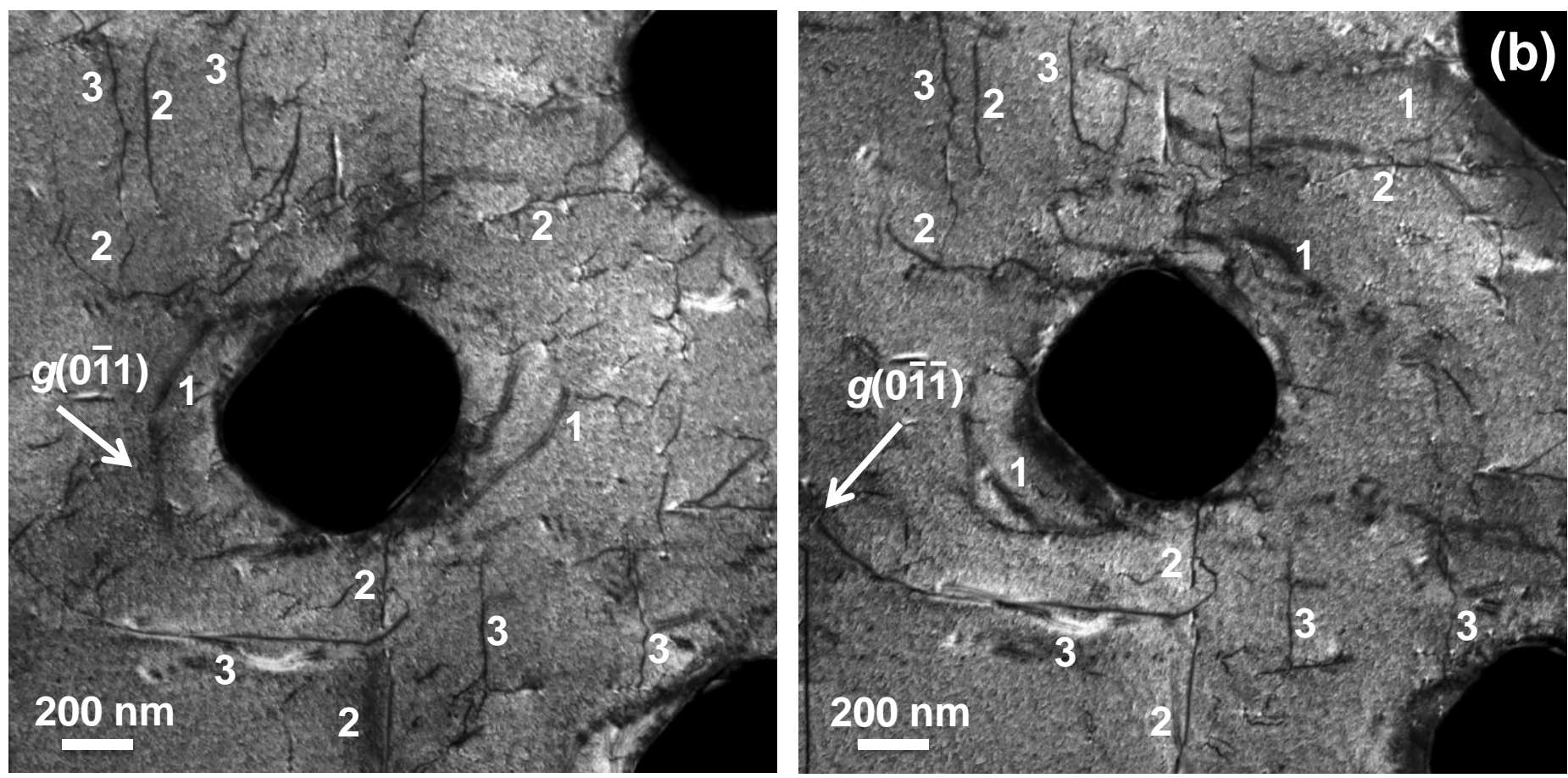

Fig. 2
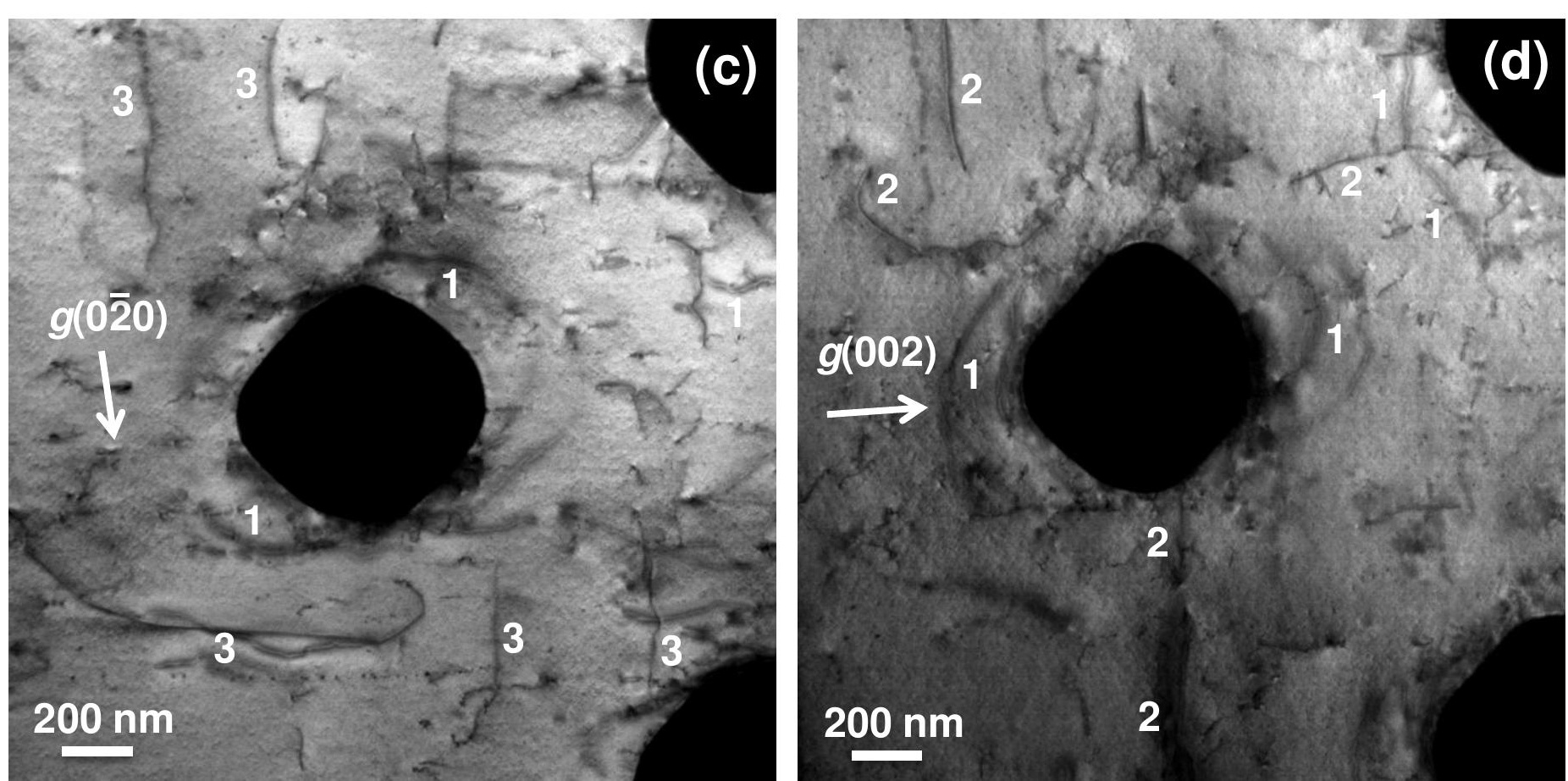

1: $b=a[100]$

2: $b=a[001]$

3: $b=a[010]$ 

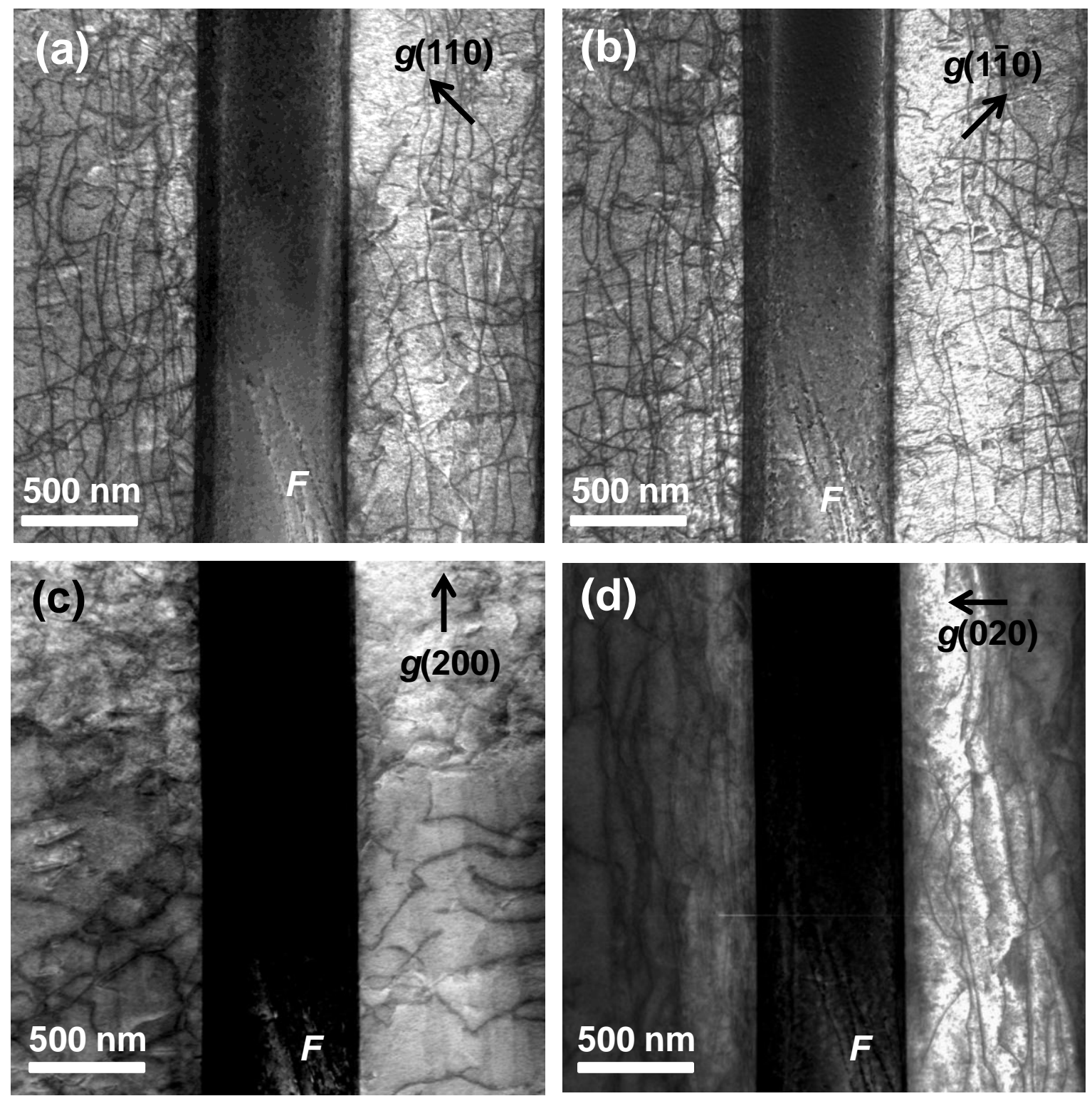

Fig. 3 


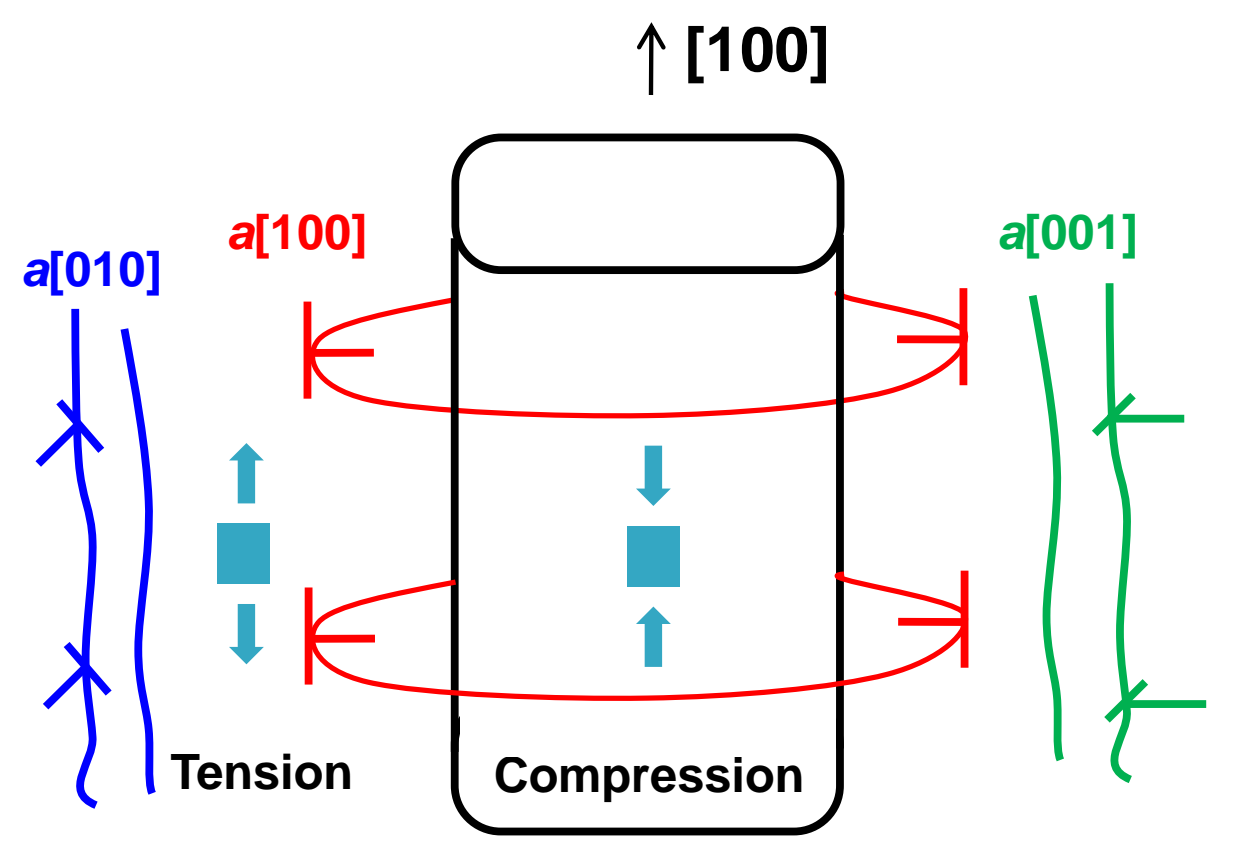

Fig. 4 

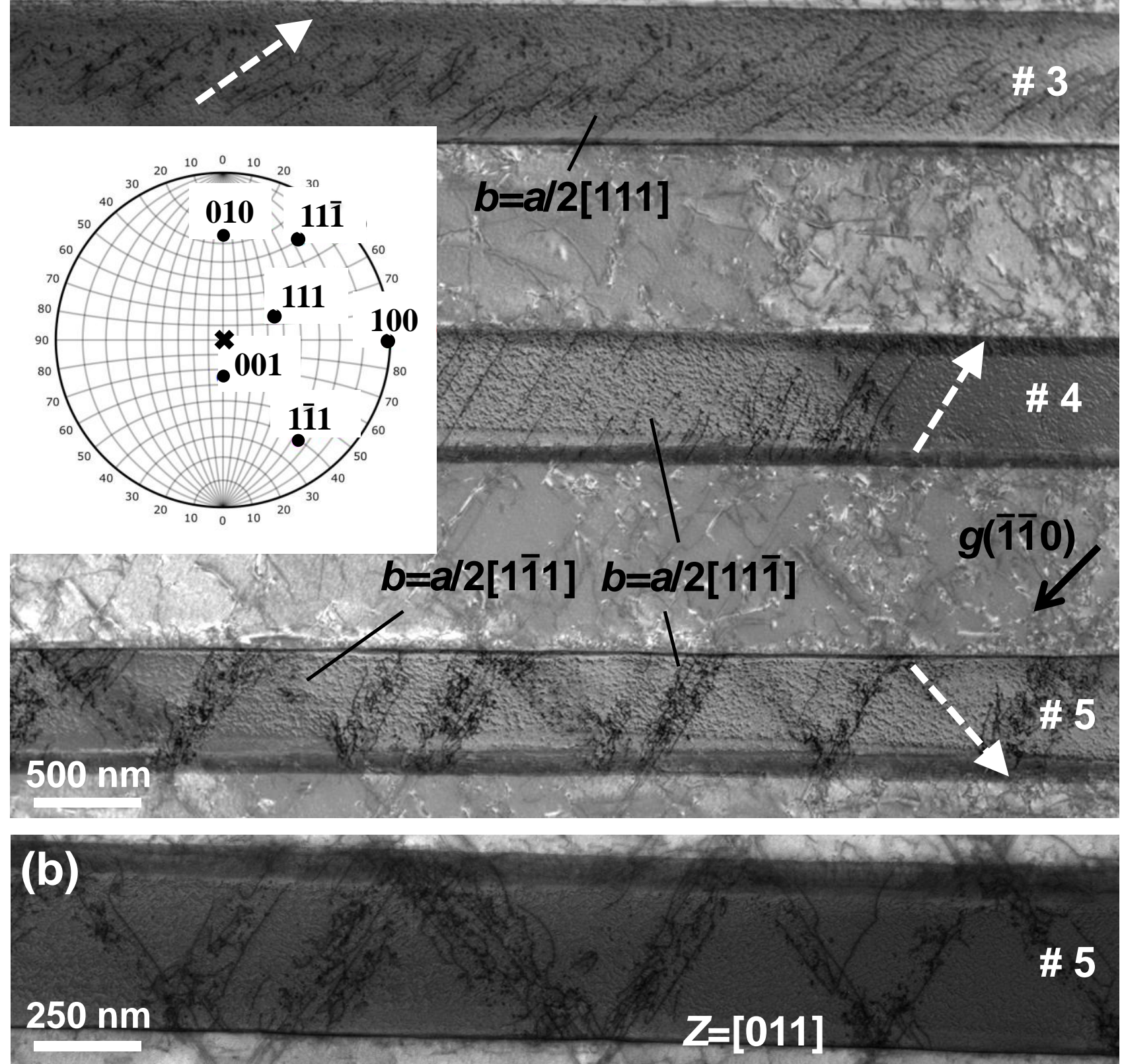

Fig. 7 


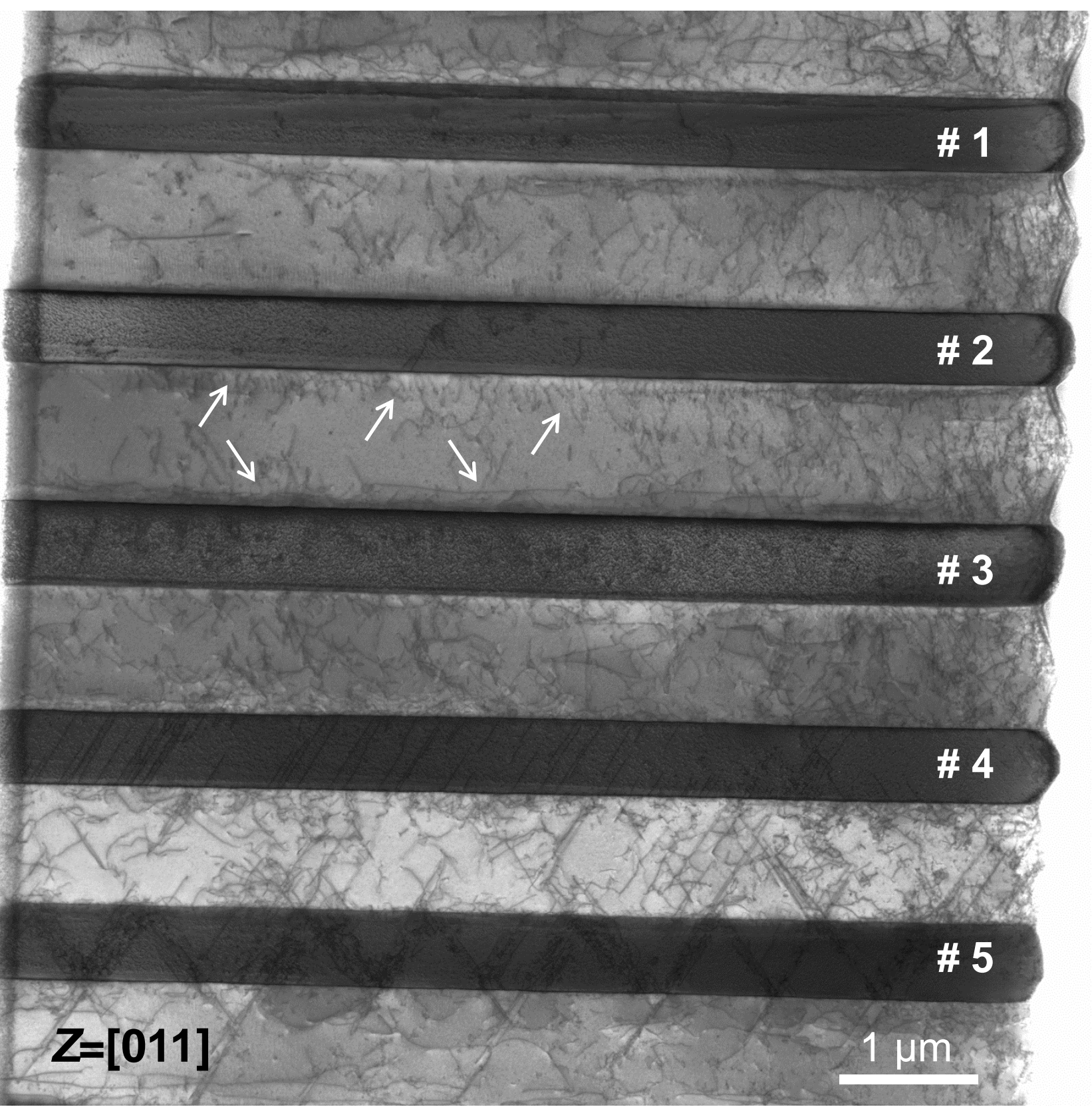

Fig. 8 


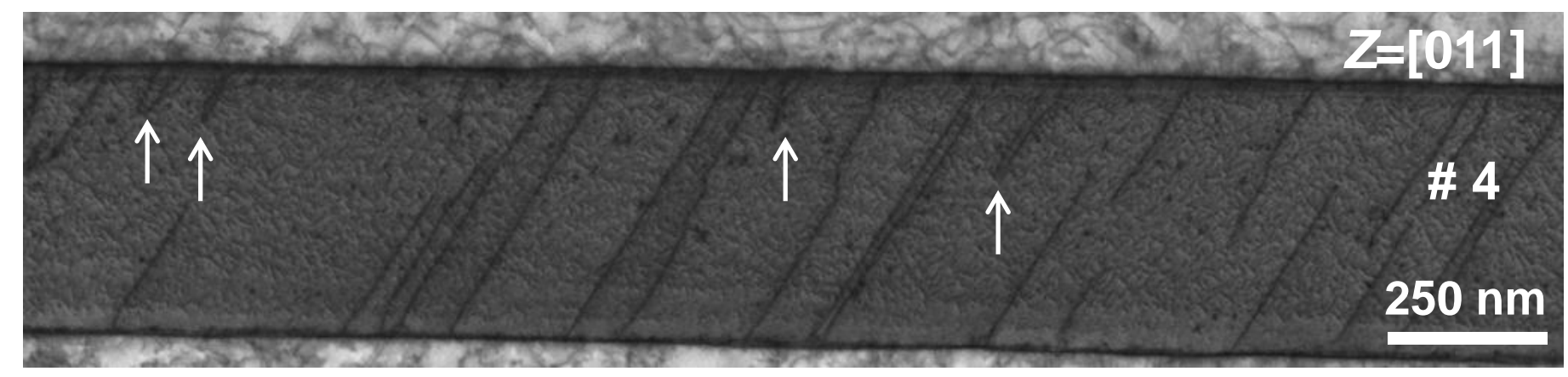

Fig. 9 


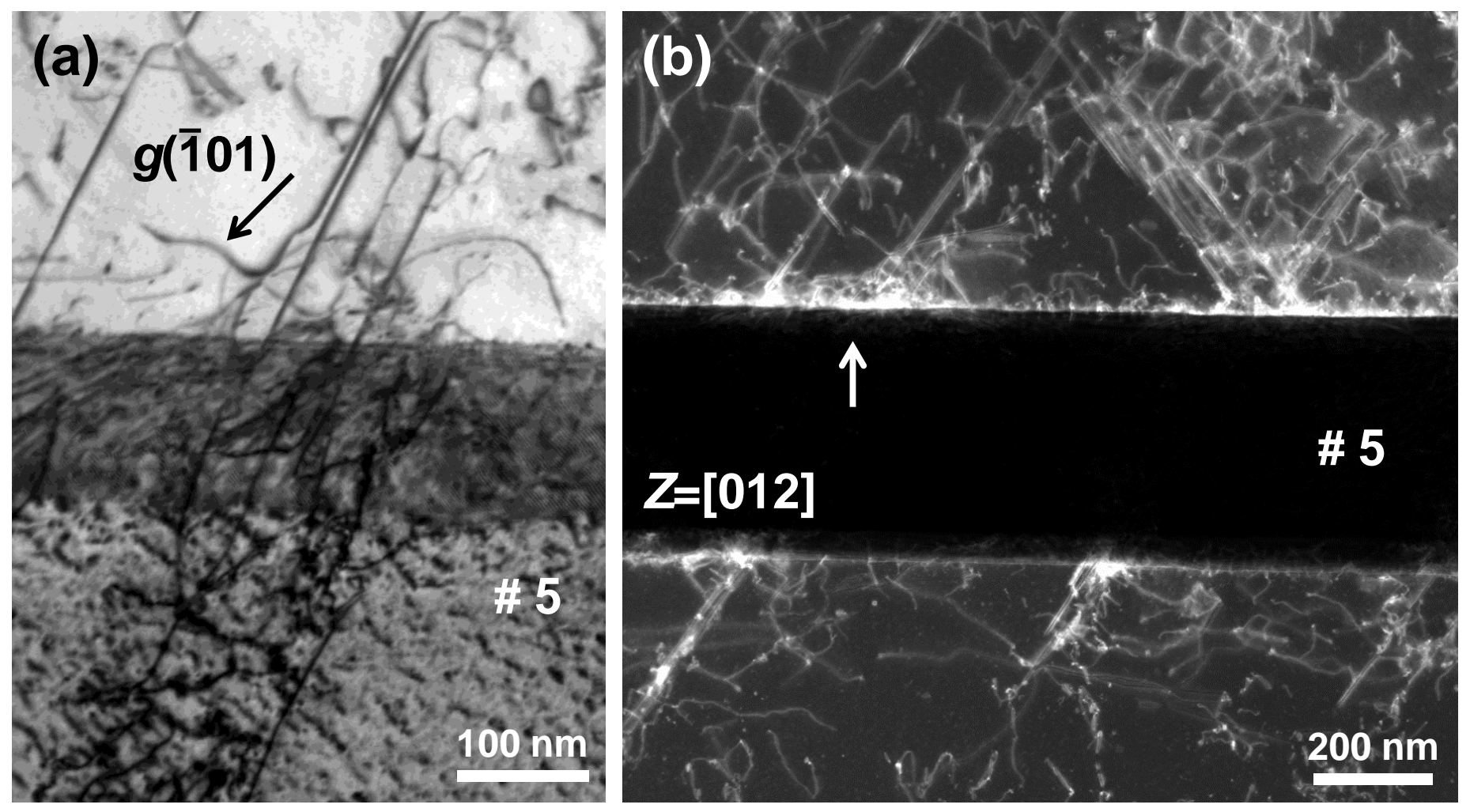

Fig. 10 

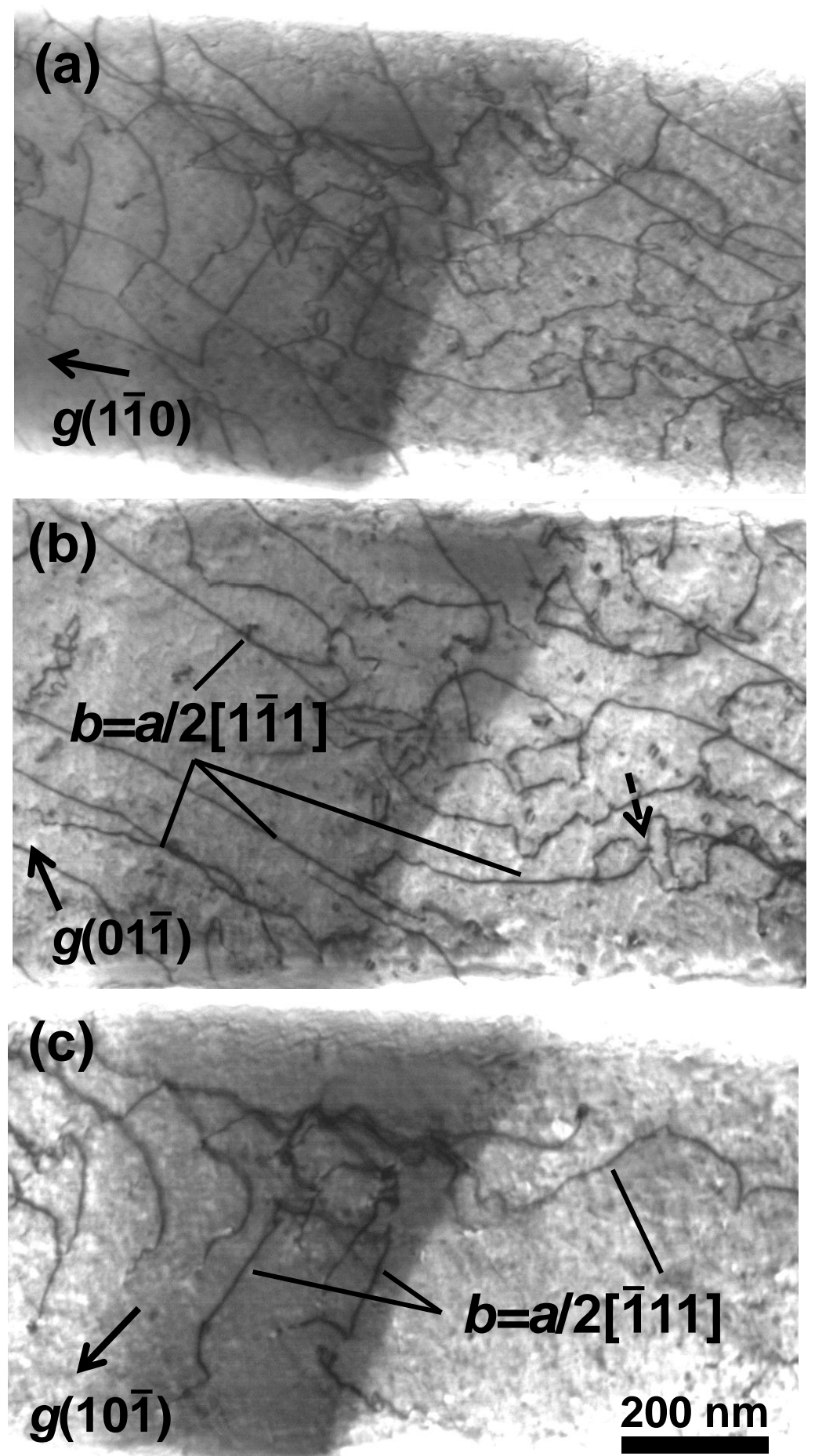

Fig. 12 
(a)

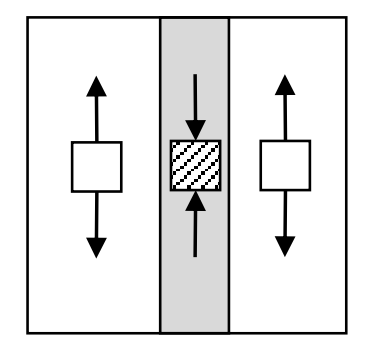

As-grown state (b)

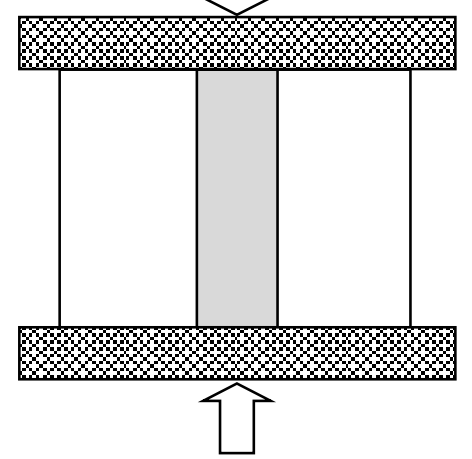

Pre-strain (c)

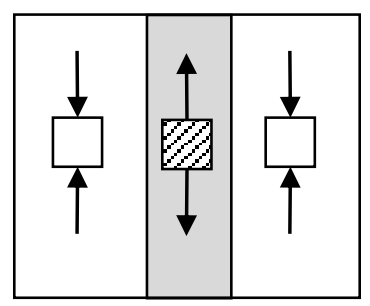

Residual stress after unloading 


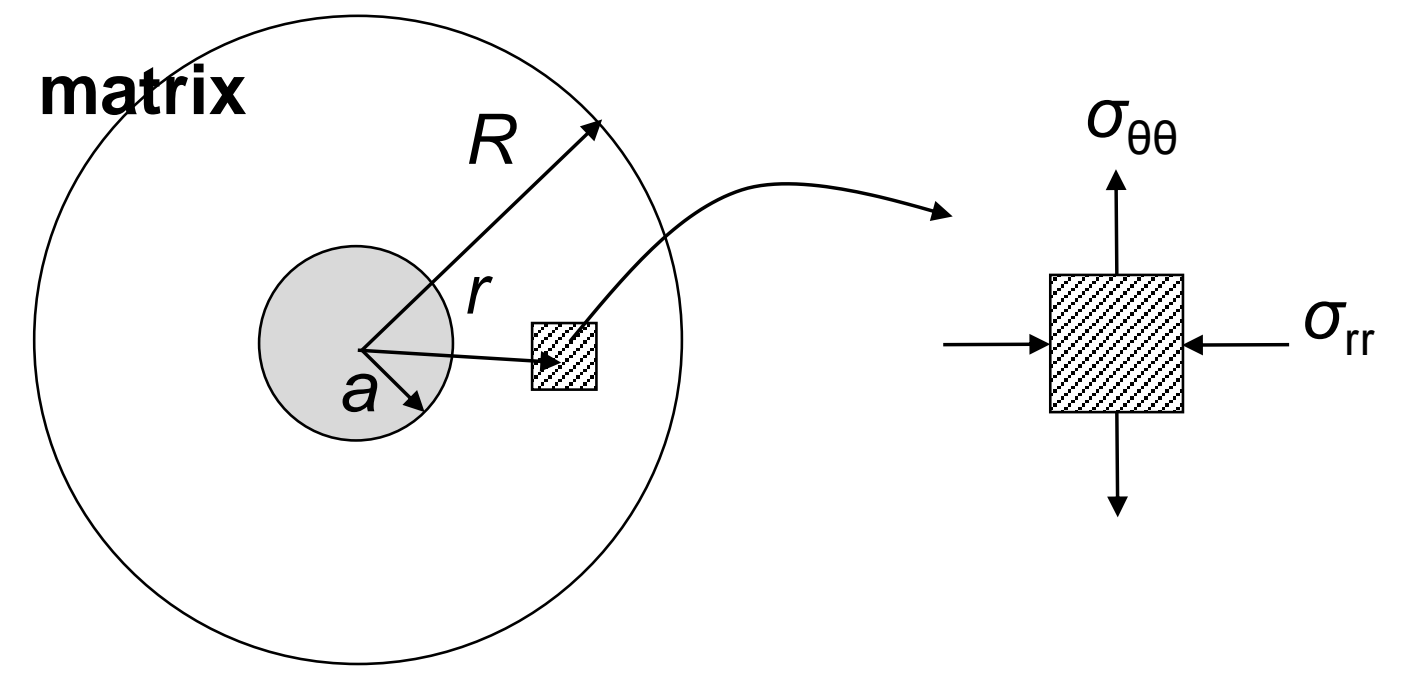

Fig. 14 


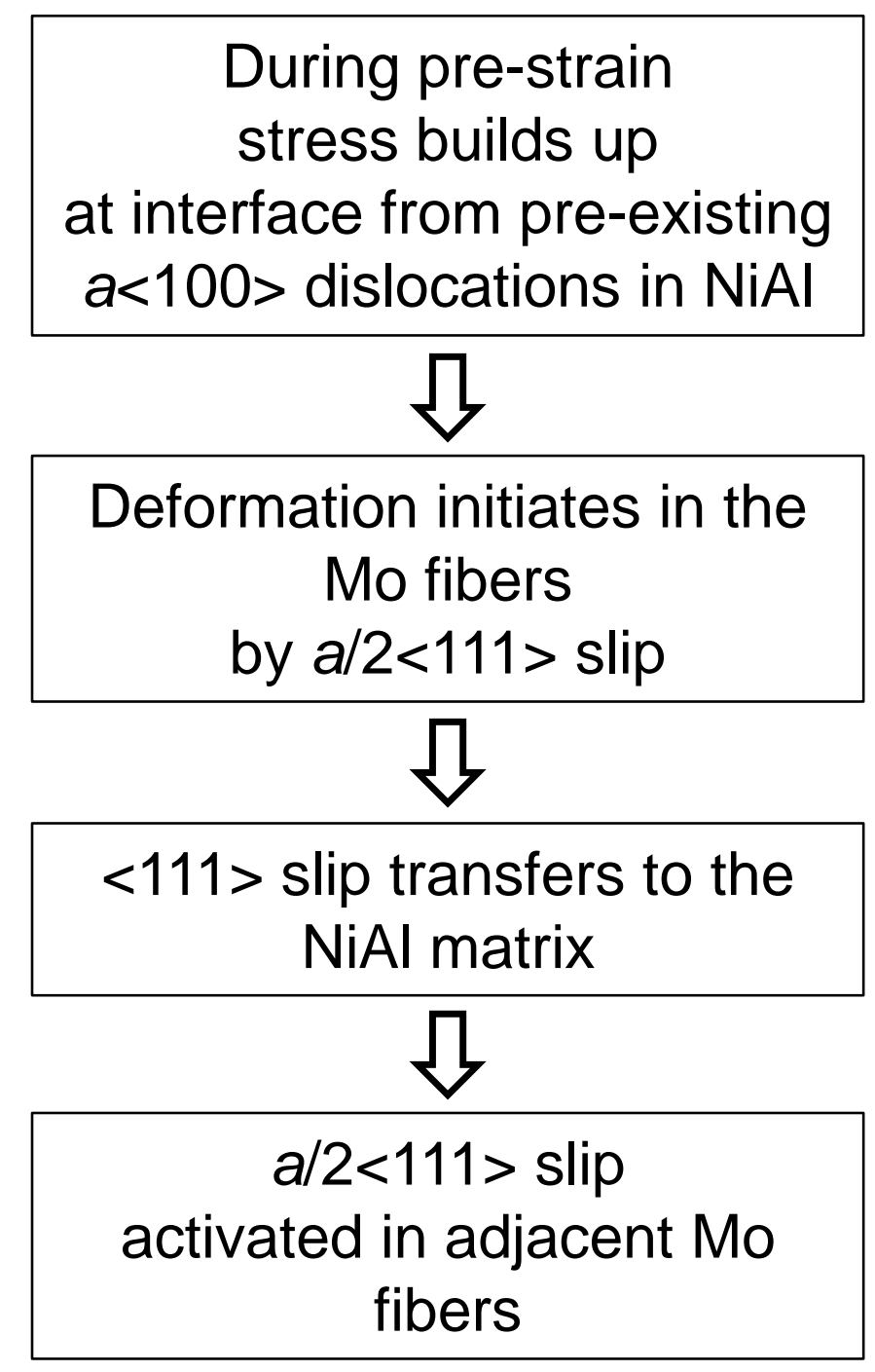

Fig. 15 